\title{
Bone Involvement in Atypical Teratoid/Rhabdoid Tumors of the CNS
}

\author{
M. Warmuth-Metz, B. Bison, N.U. Gerber, T. Pietsch, M. Hasselblatt, and M.C. Frühwald
}

\begin{abstract}
SUMMARY: Destruction of the bony structures of the skull is rare in primary tumors of the CNS. In low-grade gliomas, modeling of the skull is caused by slow growth and chronic pressure. Bony destruction is exceptional even in highly malignant gliomas. Atypical teratoid/ rhabdoid tumors of the CNS are highly malignant neoplasms diagnosed with an increasing frequency, mainly in young children. On imaging, these tumors exhibit distinct though not specific morphologic features including peripheral cysts, bleeding residues, and a distinct bandlike, wavy pattern of enhancement. A combination of these single characteristics together with a predilection for young age is suggestive of an atypical teratoid/rhabdoid tumor. We present 5 children with an atypical teratoid/rhabdoid tumor affecting the adjacent bone. These 5 patients were collected in our imaging data base for childhood atypical teratoid/rhabdoid tumor consisting of 91 children at the time of this evaluation and thus representing $6.6 \%$. The mean age of children with bone involvement (4.8 years) was above the average age (2 years) of all children in the data base. We add this rare feature to the list of typical features in MR imaging and CT morphology of atypical teratoid/rhabdoid tumor.
\end{abstract}

ABBREVIATION: AT/RT = atypical teratoid/rhabdoid tumor

$\mathbf{N}$ euroepithelial tumors rarely demonstrate destruction of adjacent skull or skull base. ${ }^{1-5}$ Atypical teratoid/rhabdoid tumors (AT/RT) are highly malignant primary tumors of the CNS, mostly occurring in very young infants. ${ }^{6}$ The incidence in children below the age of 1 year is nearly reaching the incidence of primitive neuroectodermal tumors/medulloblastomas (primitive neuroectodermal tumor/medulloblastoma). Indeed, in some series of children below 6 months of age, AT/RT has been shown to be the most common malignant CNS tumor. ${ }^{7,8}$ The genetic hallmark of rhabdoid tumors is mutation of the tumor suppressor gene SMARCB1; this results in loss of INI1 protein expression, which can be detected in most AT/RTs. ${ }^{9}$ A few cases showing

Received December 12, 2012; accepted after revision January 15, 2013.

From the National Reference Center for Neuroradiology (M.W.-M., B.B.), Department of Neuroradiology, University Hospital of Wurzburg, Wurzburg, Germany; Department of Pediatric Oncology (N.U.G.), Children's University Hospital of Zurich, Zurich, Switzerland; National Reference Center for Neuropathology (T.P.), Department of Neuropathology, University Hospital, Bonn, Germany; Institute of Neuropathology (M.H.), University Hospital Münster, Münster, Germany; and EURHAB Center of Competence for Rhabdoid Tumours (M.C.F.), Children's Hospital Augsburg, Suabian Children's Cancer Center, Augsburg, Germany, and Department of Pediatric Hematology and Oncology, University Children's Hospital Münster, Münster, Germany.

Please address correspondence to Monika Warmuth-Metz, MD, Abteilung fur Neuroradiologie, Uniklinikum Wurzburg, Josef-Schneider-Str 11, 97080 Wurzburg, Germany; e-mail: warmuth@neuroradiologie.uni-wuerzburg.de

-- Indicates open access to non-subscribers at www.ajnr.org

http://dx.doi.org/10.3174/ajnr.A3554 mutations of other chromatin remodeling complex members such as SMARCA4 have also been described. ${ }^{10}$

MR imaging features of AT/RT are described as nonspecific. $^{11-13}$ However, a combination of nonspecific singular features including bleeding residues, peripherally localized cysts, high cellularity seen as low T2 and/or ADC signal, and a distinct bandlike enhancement in a brain tumor in an infant or young child is suggestive of an AT/RT. ${ }^{12}$

We report 5 children harboring an AT/RT with skull or skull base involvement. This rare phenomenon may add more specificity to the already present list of imaging characteristics of AT/RT.

\section{MATERIALS AND METHODS}

The patients presented in the current report were collected in the National Reference Center for Neuroradiology for the German Brain Tumor (HIT) Trials, conducted in German-speaking countries of Europe. At the time of evaluation, the data base contained 91 patients diagnosed with an AT/RT. Imaging files sent to our center come from more than 100 treating institutions and are collected within multicenter studies. According to this multicenter basis of data acquisition, no uniform MR imaging protocol was applied. With the consent to participate in the trials of the HIT network, parents or patients agree to the data transfer to reference institutions. 

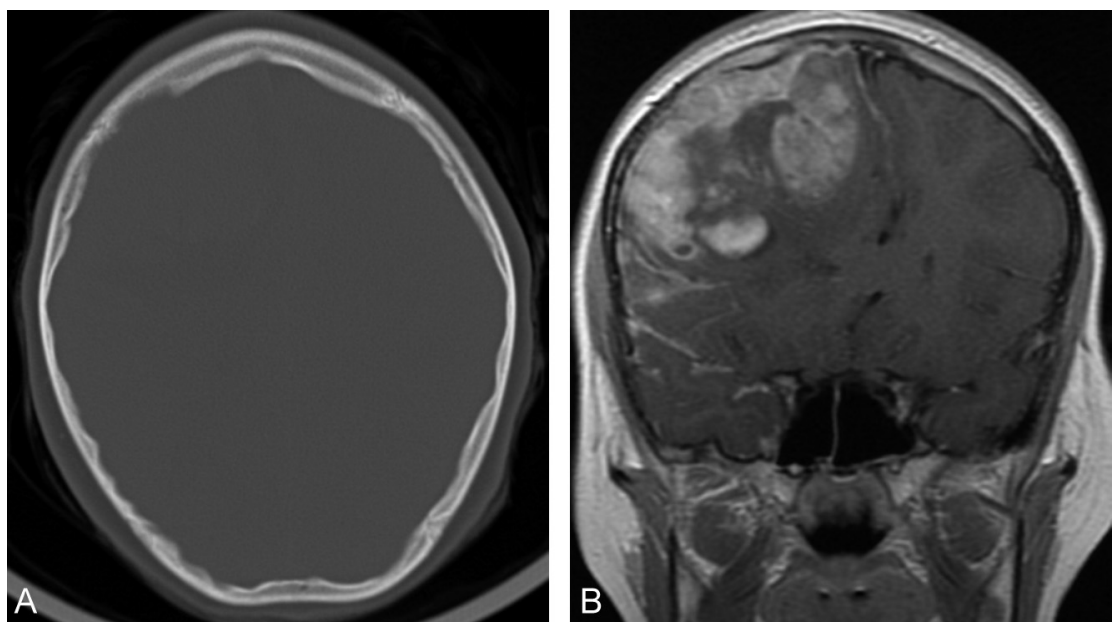

FIG 1. A, Circumscribed right frontal calvarial destruction on a CT in bone reconstruction mode. $B$, Inhomogeneously enhancing right frontal tumor showing a destruction of the inner table of the skull comparable to $A$.

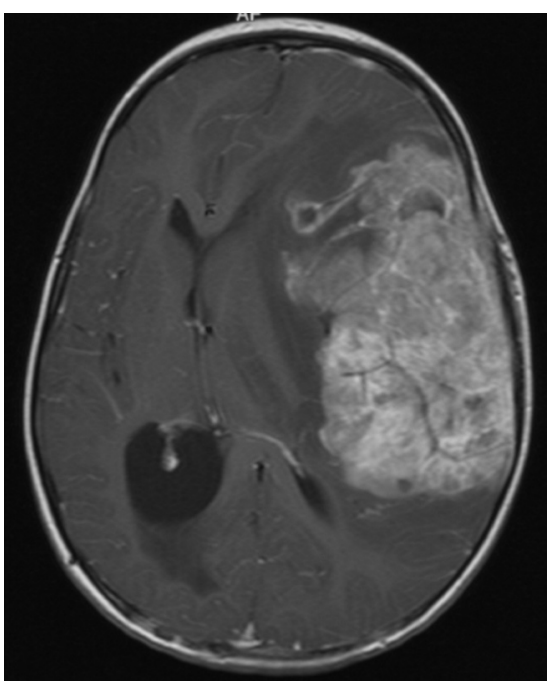

FIG 2. Contrast enhanced axial T1-weighted MRI showing a huge left hemispheric tumor with invasion of the skull and subfalcial herniation.

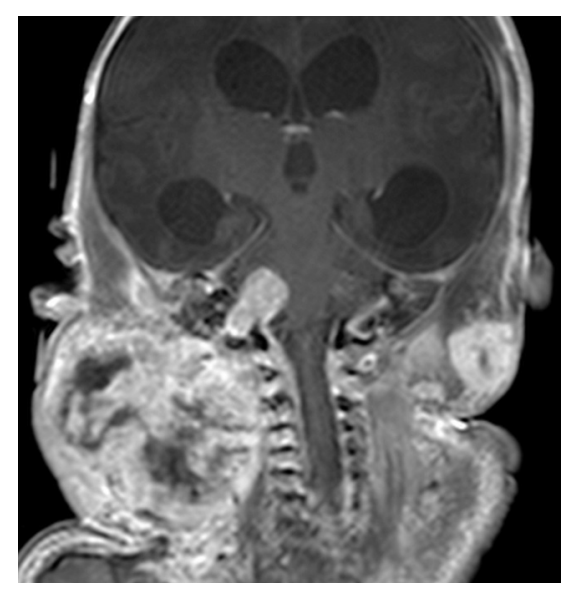

FIG 3. Coronal TI-weighted MRI after contrast showing a huge mass at the right side of the neck and an intracranial tumor with extension to the temporal bone. A smaller extracranial soft tissue tumor is seen in the left side of the neck. 16 months after diagnosis.

\section{Patient 2} months after diagnosis.

\section{Patient 3}

\section{Patient 4}

Compared with the whole group of children with an AT/RT in our data base, the 5 children presented here were older (mean age of 2 years in general; our cases, mean age of 4.8 years).

\section{Patient 1}

The 9-year-old girl presented with double vision, abducens nerve paresis, and papilledema. On MR imaging, a large tumor in the right frontal lobe was detected. The tumor demonstrated a continuous spread into the local leptomeninges but no distant leptomeningeal dissemination. The CSF was positive for tumor cells. On MR imaging and on CT, an infiltration and erosion of the overlying skull was evident (Fig 1A, $-B$ ). The patient underwent intensive systemic and intraventricular chemotherapy (Dana Farber Cancer Institute $\operatorname{protocol}^{14}$ ) and craniospinal irradiation. Six months after diagnosis, multiple brain lesions developed and an infectious complication was suspected. Biopsy of one such lesion remained inconclusive, and the patient died after local tumor progression

The 3-year-old girl presented with a huge left hemispheric tumor without leptomeningeal dissemination (Fig 2). A partial resection was performed, and chemotherapy was started. After 3 blocs of chemotherapy and before a planed stem cell transplant, the tumor progressed locally, and, after palliative treatment, the child died 6

On intrauterine MR imaging 2 days before delivery, hydrocephalic ventricles and a lesion in the right temporal lobe were seen (Fig 3). MR imaging was performed because high-risk sonography had demonstrated macrocephaly prenatally. At birth, the boy showed intracranial tumors and multiple nodules within the muscles and soft tissues of the neck, trunk, and extremities. The spleen, kidneys, pancreas, and adrenals were also affected. A biopsy of a mass affecting the upper extremity revealed a malignant rhabdoid tumor and the suspicion of synchronous rhabdoid tumors. A prognostically unfavorable rhabdoid tumor predisposition syndrome was diagnosed. After palliative chemotherapy the boy died at the age of 3 months.

The 5-year-old girl showed an extra-axially growing tumor in the cerebellopontine angle on the right side, with infiltration into the internal auditory canal (Fig 4). Preoperative hearing loss in the right ear remained unchanged after resection. The tumor was nearly totally removed, and treatment with chemotherapy and radiation therapy was completed. At the time of this evaluation, 2.5 years after diagnosis, the child is alive without disease. 

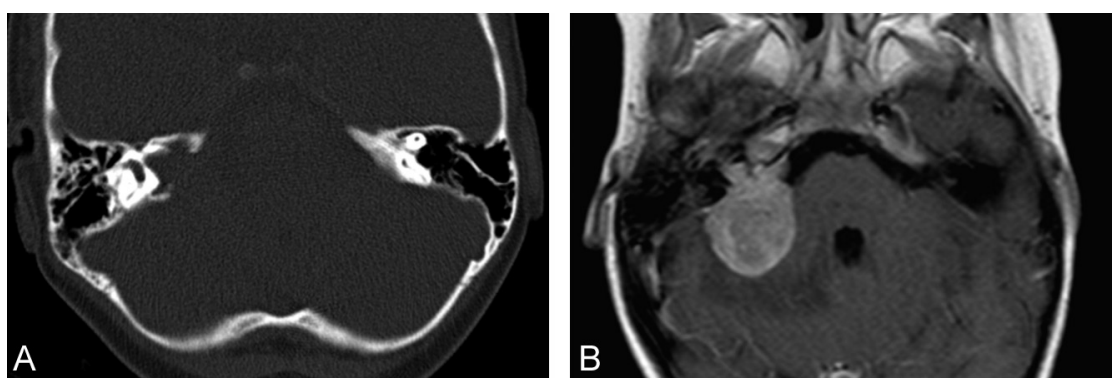

FIG 4. $A$, Bone $C T$ shows destruction around the internal acoustic canal on the right side. $B$, This tumor would resemble a vestibular schwannoma if not for the bony destruction on axial postcontrast $\mathrm{Tl}$ and previous $\mathrm{CT}$ in $\mathrm{A}$.
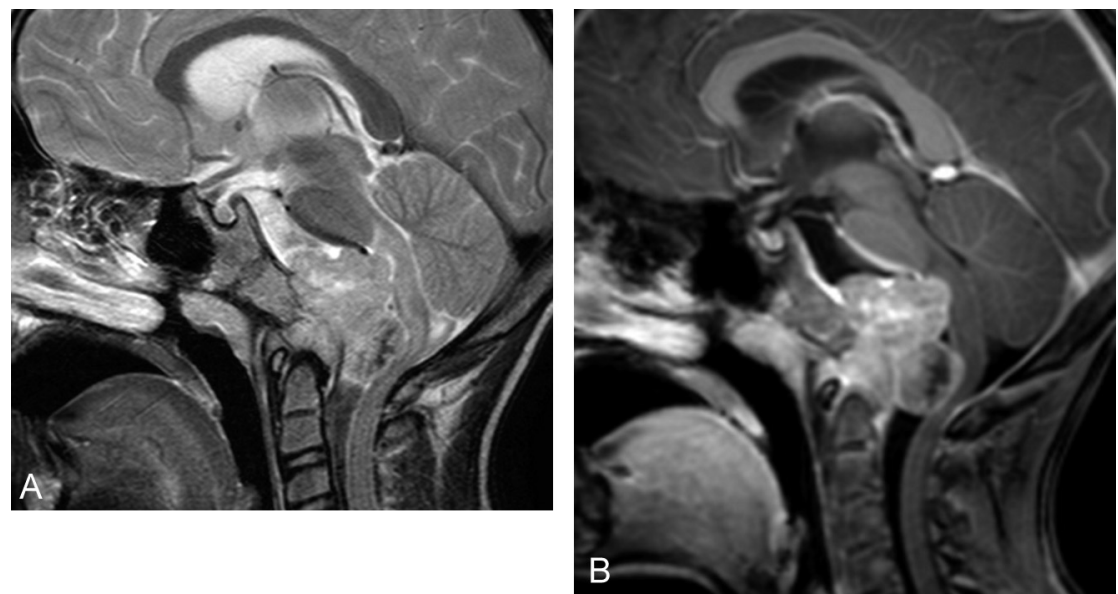

FIG 5. A, The tumor partially destroying the clivus has a relatively low T2-signal rendering a chordoma quite unlikely. B, T1-weighted contrast enhanced sagittal MRI with a lobulated solid tumor extending out of the clivus and the craniocervical junction.

\section{Patient 5}

The 7-year-old boy was diagnosed after development of nuchal pain, sickness, and general weakness. Because of destruction of the clivus and despite the low T2 signal of this tumor on initial MR imaging, a clivus chordoma was suspected (Fig 5). Biopsy revealed an AT/RT. He died 8 months after unsuccessful chemotherapy.

\section{DISCUSSION}

The dura is thought to be a natural barrier for glioma extension, rendering bone infiltration a rare event in primary brain tumors. There are only individual reports on highly malignant gliomas demonstrating growth through the untouched dura leading to calvarial destruction ${ }^{1,3-5}$ and tumor extension through the skull base into the orbit or the mouth. ${ }^{15}$ Although hematogeneous tumor spread, most frequently to the skeleton, is a rare but wellknown finding mainly in the follow-up of medulloblastomas and very rarely in gliomas, virtually no reports on a destruction or invasion of the adjacent skull in medulloblastomas or CNS primitive neuroectodermal tumors exist.

AT/RTs are highly malignant tumors of childhood. After definition of this entity, ${ }^{6}$ this tumor has been diagnosed with increasing frequency. On histopathology, rhabdoid tumor cells exhibit typical morphologic features. However, these defining cells are not found in all tumor parts, leading to problems in the discrimination of AT/RT from other embryonal tumors, for example, CNS primitive neuroectodermal tumor and medulloblastoma.
The frequent loss of the nuclear protein INI1/SMARCB1 has become state of the art for the diagnosis of intracranial rhabdoid tumor, AT/RT. ${ }^{16,17}$ Among the tumors in children below 1 year of age, AT/RT is now found nearly as frequently as CNS primitive neuroectodermal tu-

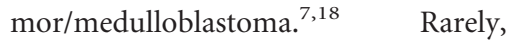
adults are diagnosed with AT/RT, which in this age group are mainly supratentorial, in contrast to young children, in whom infratentorial tumor location predominates. ${ }^{18,19}$ In addition to the high cellularity of the tumor reflected by low signal on T2-weighted and ADC images, ${ }^{20}$ bleeding residues, peripheral cysts, and a distinct pattern of contrast enhancement have been described as frequent findings. ${ }^{1-13,21}$ Rare reports of patients affected by AT/RT demonstrating involvement of the skull in children and adults exist. ${ }^{11,19}$ Two children with AT/RT of clival location (similar to our patient 5) have been described, whose radiologic appearance also led to the suspicion of chordoma despite very low signal on T2-weighted images. ${ }^{22,23}$ Indeed, in our patient, the possibility of "poorly differentiated chordoma" ${ }^{24,25}$ was also discussed during neuropathologic evaluation but rejected in favor of AT/RT, because the tumor lacked any convincing chordoid differentiation. Patient 3 does not completely fit with the other cases because he probably had multifocal malignant rhabdoid tumors of the body in addition to the probable AT/RT. Histology was not performed on the intracerebral tumor but on a peripheral tumor and revealed malignant rhabdoid tumor. The tumor leading to destruction of the petrous bone also grew outside of the skull. Therefore, we cannot exclude the possibility that this was indeed a peripheral rhabdoid tumor extending through the skull base into the brain.

The differential diagnosis of bone-eroding gliomas and AT/RT may be impossible, especially when the more frequently observed features such as cysts, bleeding residues, and very low T2 or ADC values are missing. The frequency of 5 of 91 patients with AT/RT with involvement of the skull (6.6\%) appears to be high enough to exclude the possibility of a random phenomenon and is in line with reports of AT/RT showing calvarial or skull base affection. We thus add another extraordinary imaging feature to the existing ones to facilitate the diagnosis of AT/RT.

\section{ACKNOWLEDGMENTS}

This work was supported by the Deutsche Kinderkrebsstiftung (German Childhood Cancer Foundation).

Disclosures: Monika Warmuth-Metz: RELATED: Grant: Deutsche Kinderkrebsstiftung German Childhood Cancer Foundation (Parents' association), ${ }^{*}$ Support for Travel to Meetings for the Study or Other Purposes: Deutsche Kinderkrebsstiftung German

AJNR Am J Neuroradiol 34:2039-42 Oct 2013 www.ajnr.org 
Childhood Cancer Foundation; UNRELATED: Board Membership: Roche, Grants/ Grants Pending: Deutsche Kinderkrebsstiftung German Childhood Cancer Foundation*; Travel/Accommodations/Meeting Expenses Unrelated to Activities Listed: Deutsche Kinderkrebsstiftung German Childhood Cancer Foundation, Brigitte Bison: OTHER RELATIONSHIPS: My position is paid by the German Children's Cancer Foundation, which is a parents' association collecting money to support all scientific activities of the German HIT Studies. Martin Hasselblatt: RELATED: Grant: Interdisciplinary Centre for Clinical Research, University Munster," ( ${ }^{*}$ money paid to institution).

\section{REFERENCES}

1. Gheyi V, Hui FK, Doppenberg EM, et al. Glioblastoma multiforme causing calvarial destruction: an unusual manifestation revisited. AJNR Am J Neuroradiol 2004;25:1533-37

2. Murphy MN, Korkis JA, Robson FC, et al. Gliosarcoma with cranial penetration and extension to the maxillary sinus. J Otolaryngol 1985;14:313-16

3. Osborn RE, Ley CE. Astrocytoma with calvarial erosion. AJNR Am J Neuroradiol 1986;7:178

4. Schuss P, Ulrich CT, Harter PN, et al. Gliosarcoma with bone infiltration and extracranial growth: case report and review of literature. J Neuro-oncol 2011;103:765-70

5. Woodruff WW Jr, Djang WT, Voorhees D, et al. Calvarial destruction: an unusual manifestation of glioblastoma multiforme. AJNR Am J Neuroradiol 1988;9:388-89

6. Rorke LB, Packer RJ, Biegel JA. Central nervous system atypical teratoid/rhabdoid tumors of infancy and childhood: definition of an entity. J Neurosurg 1996;85:56-65

7. von Hoff K, Hinkes B, Dannenmann-Stern E, et al. Frequency, riskfactors and survival of children with atypical teratoid rhabdoid tumors (AT/RT) of the CNS diagnosed between 1988 and 2004, and registered to the German HIT database. Pediatr Blood Cancer 2011;57:978-85

8. Woehrer A, Slavc I, Waldhoer T, et al. Incidence of atypical teratoid/ rhabdoid tumors in children: a population-based study by the Austrian Brain Tumor Registry, 1996-2006. Cancer 2010;116:5725-32

9. Jackson EM, Sievert AJ, Gai X, et al. Genomic analysis using highdensity single nucleotide polymorphism-based oligonucleotide arrays and multiplex ligation-dependent probe amplification provides a comprehensive analysis of INI1/SMARCB1 in malignant rhabdoid tumors. Clin Cancer Res 2009;15:1923-30

10. Schneppenheim R, Fruhwald MC, Gesk S, et al. Germline nonsense mutation and somatic inactivation of SMARCA4/BRG1 in a family with rhabdoid tumor predisposition syndrome. Am J Hum Genet 2010;86:279-84

11. Arslanoglu A, Aygun N, Tekhtani D, et al. Imaging findings of CNS atypical teratoid/rhabdoid tumors. AJNR Am J Neuroradiol 2004; 25:476-80

12. Warmuth-Metz M, Bison B, Dannemann-Stern E, et al. CT and MR imaging in atypical teratoid/rhabdoid tumors of the central nervous system. Neuroradiology 2008;50:447-52

13. Cheng YC, Lirng JF, Chang FC, et al. Neuroradiological findings in atypical teratoid/rhabdoid tumor of the central nervous system. Acta Radiologica 2005;46:89-96

14. Chi SN, Zimmerman MA, Yao X, et al. Intensive multimodality treatment for children with newly diagnosed CNS atypical teratoid rhabdoid tumor. J Clin Oncol 2009;27:385-89

15. Horiuchi T, Osawa M, Itoh N, et al. Extradural extension of glioblastoma multiforme into the oral cavity: case report. Surg Neurol 1996;46:42-46

16. Judkins AR, Mauger J, Ht A, et al. Immunohistochemical analysis of hSNF5/INI1 in pediatric CNS neoplasms. Am J Surg Pathol 2004;28:644-50

17. Miller S, Ward JH, Rogers HA, et al. Loss of INI1 protein expression defines a subgroup of aggressive central nervous system primitive neuroectodermal tumors. Brain Pathol 2013;23:19-27

18. Takahashi K, Nishihara H, Katoh M, et al. Case of atypical teratoid/ rhabdoid tumor in an adult, with long survival. Brain Tumor Pathol 2011;28:71-76

19. Han L, Qiu Y, Xie C, et al. Atypical teratoid/rhabdoid tumors in adult patients: CT and MR imaging features. AJNR Am J Neuroradiol 2011;32:103-08

20. Yamashita Y, Kumabe T, Higano S, et al. Minimum apparent diffusion coefficient is significantly correlated with cellularity in medulloblastomas. Neurol Res 2009;31:940-46

21. Parmar $\mathrm{H}$, Hawkins $\mathrm{C}$, Bouffet $\mathrm{E}$, et al. Imaging findings in primary intracranial atypical teratoid/rhabdoid tumors. Pediatr Radiol 2006;36:126-32

22. Heuer GG, Kiefer H, Judkins AR, et al. Surgical treatment of a clival-C2 atypical teratoid/rhabdoid tumor. J Neurosurg Pediatr 2010;5:75-79

23. Kazan S, Goksu E, Mihci E, et al. Primary atypical teratoid/rhabdoid tumor of the clival region: case report. J Neurosurg 2007;106(4 Suppl):308-11

24. Mobley BC, McKenney JK, Bangs CD, et al. Loss of SMARCB1/INI1 expression in poorly differentiated chordomas. Acta Neuropathol 2010;120:745-53

25. Hoch BL, Nielsen GP, Liebsch NJ, et al. Base of skull chordomas in children and adolescents: a clinicopathologic study of 73 cases. $\mathrm{Am} \mathrm{J}$ Surg Pathol 2006;30:811-18 